\title{
Quantitative Evaluation of Defect Based on Ultrasonic Guided Wave and CHMM
}

\author{
Le Chen, Yuemin Wang, Haiquan Geng, Wenli Deng1 and Wei Ye \\ Power Engineering College, Naval University of Engineering, 430033 Wuhan, China
}

\begin{abstract}
The axial length of pipe defects is not linear with the reflection coefficient, which is difficult to identify the axial length of the defect by the reflection coefficient method. Continuous Hidden Markov Model (CHMM) is proposed to accurately classify the axial length of defects, achieving the objective of preliminary quantitative evaluation. Firstly, wavelet packet decomposition method is used to extract the characteristic information of the guided wave signal, and Kernel Sliced Inverse Regression (KSIR) method is used to reduce the dimension of feature set. Then, a variety of CHMM models are trained for classification. Finally, the trained models are used to identify the artificial corrosion defects on the outer surface of the pipe. The results show that the CHMM model has better robustness and can accurately identify the axial defects.
\end{abstract}

\section{Introduction}

Guided wave technology is a kind of efficient detection technology, which has a great advantage in the detection of long distance pipeline, and it has been widely used in engineering. The defect size is generally judged according to the amplitude of the defect echo in practical test. We can obtain more structural information when further processing of the guided wave signal, and it's helpful to judge the healthy state of the structure more accurately.

Many scholars have conducted a lot of research on the quantitative analysis of the defects based on the guided wave signals. Demma [1] put forward a method of calculating the reflection coefficient of the notch by studying the SHO guide wave on plate, and pointed out that the reflection coefficients and defect length are not monotonically increasing relation. Peter [2] proposed a matching pursuit method to decompose the front and back signal of the defect, and estimated the defect size by comparing the differences of the decomposed signal.

Hidden Markov model (HMM) is applied in speech signal recognition in the early, and introduced into the fault diagnosis field [3]. The HMM and fault diagnosis are the same in essence, that is to say, the true state is "hidden", which can be detected only through observation vector. Therefore, the application of HMM in the field of fault diagnosis is feasible.

The raw data of pipeline axial defects is obtained by the longitudinal guided wave technique, and the characteristics are extracted using wavelet packet decomposition. The KSIR method is proposed to reduce the dimension of feature data. Finally, the CHMM classified model is applied to the recognition of the defect axial length, achieving the preliminary quantification.

\section{Theory Fundament}

\subsection{Theory of CHMM}

In contrast to the conventional HMM, the observed value of CHMM (Continuous CHMM) is continuous, which is generally assumed to be generated by Gauss PDF (probability density function). In practical applications, the assumption of Gauss PDF is often unable to meet the requirements, so the linear combination of several Gauss PDFs is used to simulate the observation sequence. The combination of a sufficient number of Gauss PDFs can approximate any PDF, and the mean and covariance matrix of each Gauss PDF can be obtained by the statistics of a large number of observation samples.

HMM can be expressed as $\lambda=(\pi, A, B)$, where $\pi$ for the initial probability matrix, $A$ for the state of transtionprobablity matrix, $B$ for the probability matrix of the observation. In CHMM, $B$ is a set of probability density functions, $B=\left\{b_{j o}\right\}$ :

$$
\begin{aligned}
b_{j \mathbf{0}} & =\sum_{k=1}^{M} c_{j k} N\left(\mathrm{O}, \mu_{j k}, U_{j k}\right) \\
& =\sum_{k=1}^{M} \frac{c_{j k}}{(2 \pi)^{D / 2}\left|U_{j k}\right|^{1 / 2}} \exp \left[-\frac{\left(o-\mu_{j k}\right)\left(o-\mu_{j k}\right)^{T}}{2 U_{j k}}\right]
\end{aligned}
$$

The significance of each parameter in the formula (1) is as follows:

$M$ : the number of Gauss probability density functions used to fit the distribution. 
$c_{j k}$ : the weight coefficients of the $k$-th Gauss probability density function in state $S_{j}$.

$N$ : Gauss probability density function.

$\mathrm{O}$ : observation sequence.

$\mu_{j k}$ : the mean vector of the $k$-th Gauss probability density function in state $S_{j}$.

$U_{j k}$ : the covariance matrix of the $k$-th Gauss PDF in state $S_{j}$.

Therefore, CHMM can be represented as $\lambda=(\pi, A, C, \mu, U)$. The training algorithm of CHMM can refer to $[4,5]$

\subsection{KSIR - dimension reduction method}

Sliced Inverse Regression (SIR) is an effective method to reduce the dimension of data, which is proposed by $\mathrm{Li}$ [6]. There is a non-linear relationship between the physical characteristics, which limits the application of SIR. Kernel Sliced Inverse Regression (KSIR) [7] can adapt to the nonlinear relationship and obtain higher classification accuracy with less feature vectors. The basic concept of KSIR is to map the samples to higher dimensional space by a nonlinear transformation, and then to calculate the inverse of the slices. That is:

$$
\Phi: x_{i} \rightarrow \phi\left(x_{i}\right)
$$

Where $\phi\left(x_{i}\right)$ belongs to a higher dimensional space than $R^{p}$.

The model $y=g\left(X \beta_{1}, X \beta_{2}, \cdots, X \beta_{M}, \varepsilon\right)$ proposed by $\mathrm{Li}$ is changed to

$$
y=g\left(\Phi(X) \beta_{1}, \Phi(X) \beta_{2}, \cdots, \Phi(X) \beta_{M}, \varepsilon\right)
$$

$\left(\beta_{1}, \beta_{2}, \cdots, \beta_{M}\right)$ is called the effective dimension reduction space, and each vector $\beta_{m}$ is effective dimension reduction direction. The calculation procedure of KSIR is as follows:

1. Standardize $\Phi(X)$ to meet

$$
\sum_{i=1}^{n} \Phi\left(x_{i}\right)=0
$$

The covariance matrix

$$
\Sigma_{x x}=\frac{1}{n} \sum_{i=1}^{n} \Phi\left(x_{i}\right) \Phi\left(x_{i}\right)^{T}
$$

2. Divide $\Phi(X)$ into $H$ slices according to the value of $y$, and the sample number of the $h$-th slice is $n_{h}$.

3. Calculate the sample mean of each slice, and the weighted covariance matrix.

$$
\bar{\Phi}_{h}=\frac{1}{n_{h}} \sum_{y(h) \in h} \Phi_{h}
$$

$$
\Sigma=\frac{1}{n} \sum_{h=1}^{H} n_{h}\left(\bar{\Phi}_{h}\right) \cdot\left(\bar{\Phi}_{h}\right)^{T}
$$

4. Calculate the eigenvalues $\lambda_{1}>\lambda_{2}>\cdots>\lambda_{p}$ and the corresponding eigenvectors $\beta_{1}, \beta_{2}, \cdots, \beta_{p}$ of the covariance matrix $\Sigma$.

5. Project the $\bar{X}$ along the first $M$ eigenvectors, and the results are $M$-dimension feature component. The selection of $M$ is based on the cumulative contribution rate of the characteristic value. In practical application, the general selection contribution rate is $80 \%-90 \%$ or higher.

\subsection{The identification process of pipe axial defect length based on the KSIR-CHMM}

To identify the axial length of pipeline defect based on CHMM, the guided wave signals are collected and the feature is extracted, and KSIR algorithm is used to reduce the dimensions of feature data for the application of CHMM. Then, the CHMM classifier is trained, which will be used to classify accurately as different axial length. The flow chart of the identification process based on the KSIR-CHMM is shown in Figure 1.

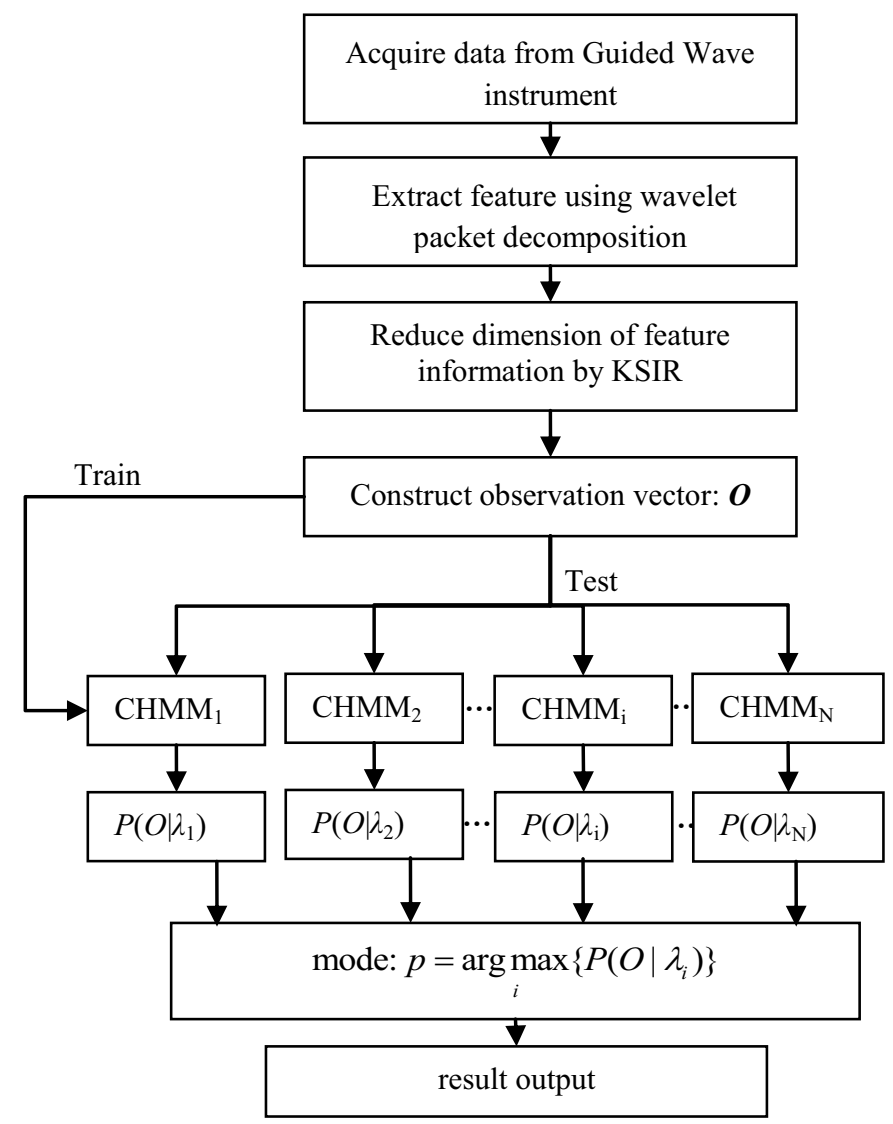

Figure 1. The identification flow chart based on KSIR-CHMM

\section{Experimental Verification}

\subsection{Experimental Setup.}


The magnetostrictive guided wave detection system is used to detect the steel pipe with an artificial corrosion on the outside surface, as shown in Figure 2, and a series of guided wave signals are recorded with the increase of defect length. The specifications of the test pipeline are shown in Table 1 . The excitation signal frequency is 53 $\mathrm{KHz}$, and the sampling frequency is $5 \mathrm{MHz}$. The signal in the dashed box as shown in Figure 3 is used for testing. Table 2 shows the samples of axial length for classification training.

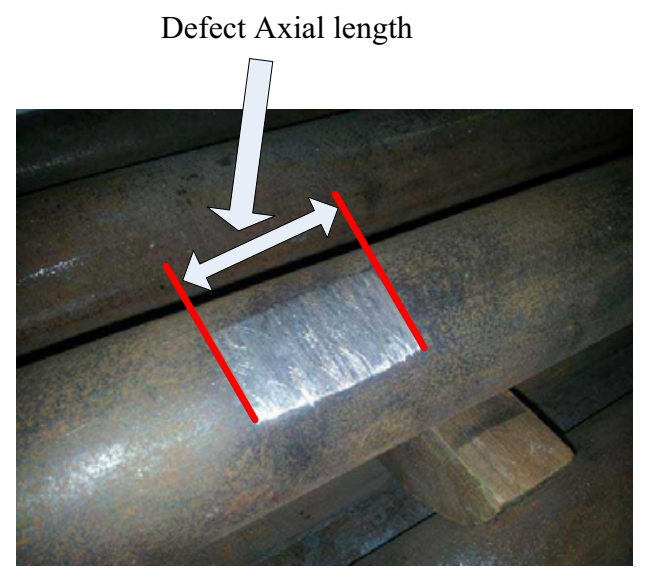

Figure 2. Photo of the artificial corrosion

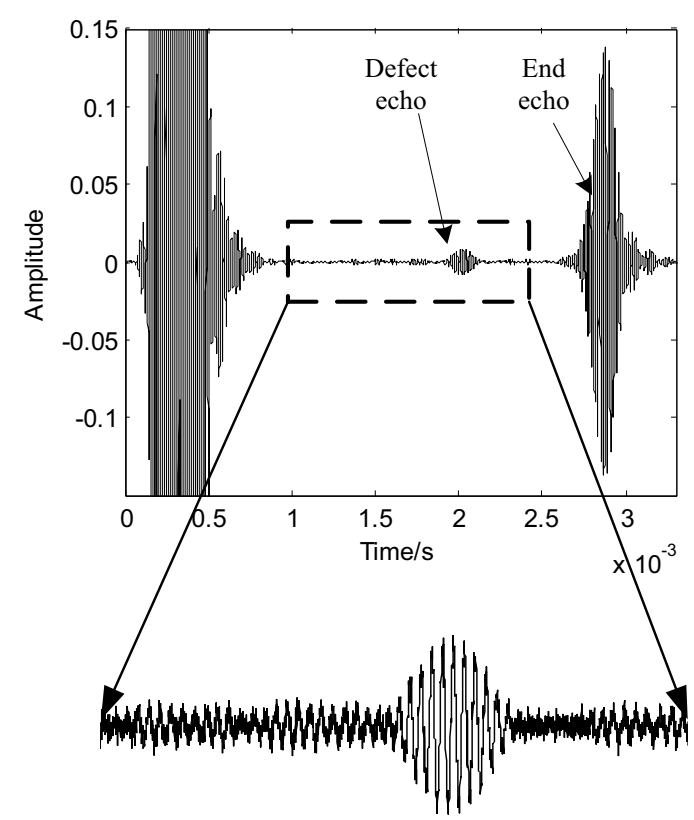

Figure 3. Testing sample signal

Table 1. Pipeline specifications to be tested

\begin{tabular}{|l|c|}
\hline \multicolumn{1}{|c|}{ Item } & Specifications $/ \mathrm{mm}$ \\
\hline Length of pipe & 6500 \\
\hline Outside diameter & 57 \\
\hline Thickness & 3.5 \\
\hline Defect position & 4333 \\
\hline Defect depth & 2.25 \\
\hline
\end{tabular}

Table 2. Samples of axial length for classification training

\begin{tabular}{|c|cc|}
\hline Damage level & Defect axial length $/ \mathbf{m m}$ \\
\hline CLASS 1 & 4.5 & 6.6 \\
\hline CLASS 2 & 9.8 & 12.5 \\
\hline CLASS 3 & 19.5 & 22.8 \\
\hline
\end{tabular}

\begin{tabular}{|l|ll|}
\hline CLASS 4 & 25.5 & 29.2 \\
\hline CLASS 5 & 35.5 & 39.8 \\
\hline
\end{tabular}

\subsection{Wavelet Packet Decomposition}

The signal is decomposed by three-layer wavelet packet, and reconstructed in 8 frequency bands. Five parameters for each band are extracted, including root mean square value, variance, skewness, kurtosis and the energy of the frequency band. Finally, a 40-dimension feature vector is constructed.

\subsection{Dimension Reduction by KSIR}

The features set are normalized before reduced. The cumulative contribution rate of $85 \%$ is chosen to determine the reduced dimension of feature. In the end, the 40-dimension feature vector is reduced to 4dimension.

\subsection{CHMM Model Training}

Figure 4 shows the training process of different CHMM classifier, and we can see that each classifier achieves convergence in less than 20 steps.

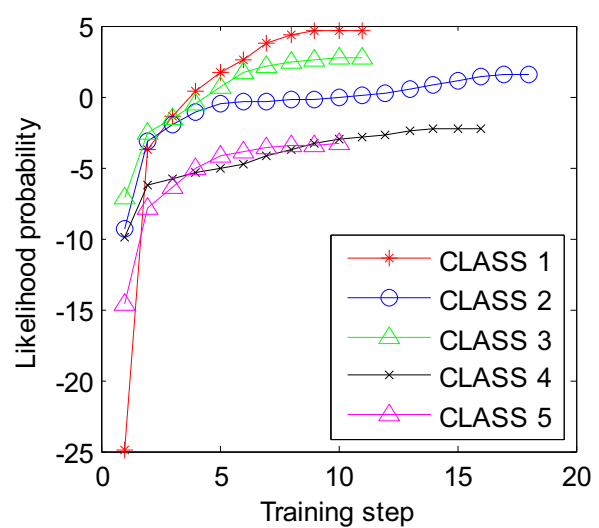

Figure 4. The training process of CHMM

\subsection{Application of CHMM Model}

Five samples with different axial length of the defect $(5 \mathrm{~mm}, 10.9 \mathrm{~mm}, 21.1 \mathrm{~mm}, 27.5 \mathrm{~mm}$ and $37.3 \mathrm{~mm}$, corresponding to CLASS 1 to CLASS 5 respectively) are used to verify the model. The identification results used CHMM are shown in Figure 5. For example, Figure 5(a) is the result of 5mm-length defect by trained CHMM, and the maximum value of likelihood probability corresponds to CLASS 1, which means the sample belongs to CLASS 1. The results show that the CHMM models can accurately identify the axial defects, and achieve the objective of preliminary quantitative evaluation. 


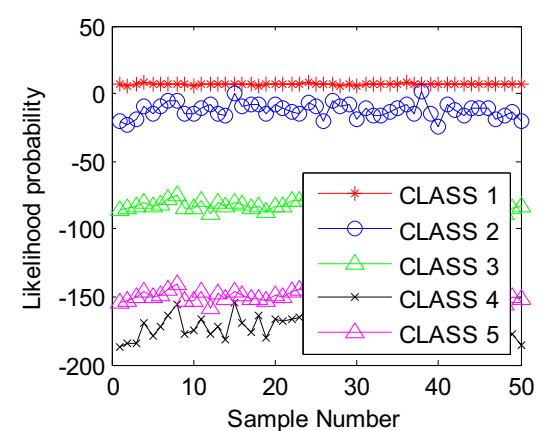

(a) $5 \mathrm{~mm}$

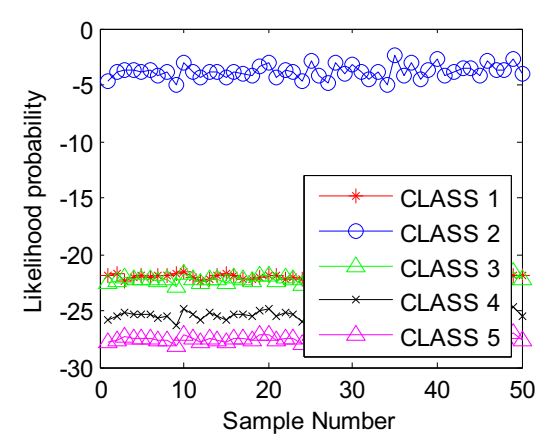

(b) $10.9 \mathrm{~mm}$

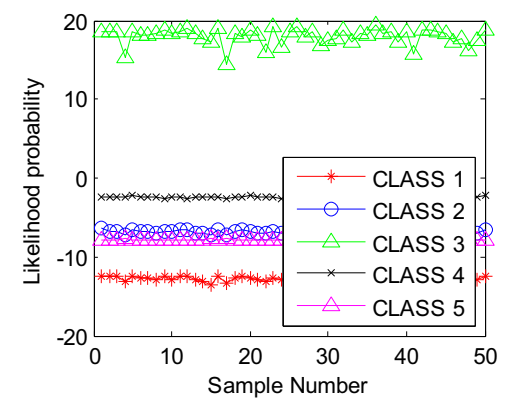

(c) $21.1 \mathrm{~mm}$

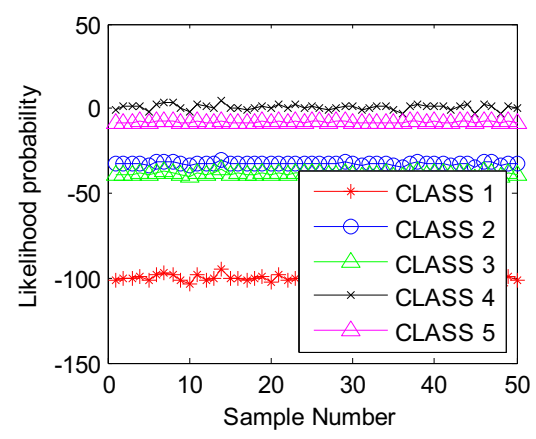

(d) $27.5 \mathrm{~mm}$

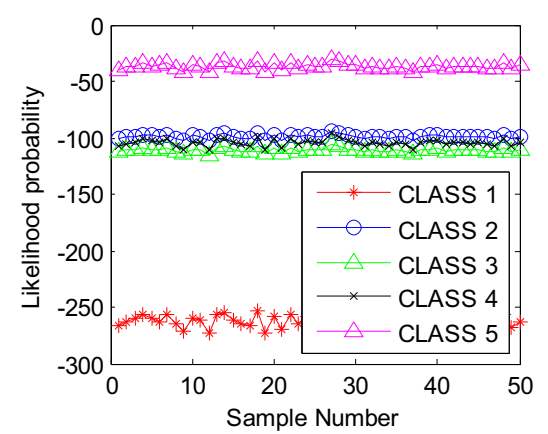

(e) $37.3 \mathrm{~mm}$

\section{Summary}

The axial length of pipe defects is not linear with the reflection coefficient, and the length of the defect cannot be judged directly by the reflection coefficient. Wavelet packet decomposition is applied to extract features, then, KSIR is used to reduce the feature dimension, and finally the CHMM model is used for classification. The following conclusions can be obtained.

1. KSIR can be used to reduce the dimension of feature matrix, which will be helpful to reduce the computation time and improve the accuracy in classification process.

2. The CHMM model can effectively distinguish the different axial length of defects, and the state is given in the form of likelihood probability. The experimental results show that the method has good practicability in pipeline health assessment.

\section{References}

1. A. Demma, P. Cawley and M. Lowe, J.Acoust. Soc. Am., 113 (2003).

2. $\quad$ P. W. Tse and X. Wang, NDT \& E Int., 54 (2013).

3. L. Y. Zhao, L. Wang and R. Q. Yan, Entropy, 17 (2015)

4. L. R. Rabiner, B. H. Juang, S. E. Levinson, and M. M. Sondhi, At \& T Technical Journal, 64 (1985).

5. J. M. Lee, S. Kim, Y. Hwang, and C. Song, J. Sound. Vib., 276 (2004).

6. K. C. Li, J. Am. Stat. Assoc., 86 (1991).

7. H. M. Wu, J. Comput. Graph. Stat., 17 (2008). 